\title{
High Seroprevalence of Leptospira Exposure in Meat Workers in Northern Mexico: A Case-Control Study
}

\author{
Cosme Alvarado-Esquivel ${ }^{\mathrm{a}, \mathrm{d}}$, Jesus Hernandez-Tinoco ${ }^{\mathrm{b}}$, Luis Francisco Sanchez-Anguiano ${ }^{\mathrm{b}}$, \\ Agar Ramos-Nevarez ${ }^{c}$, Sandra Margarita Cerrillo-Soto ${ }^{c}$, Leandro Saenz-Soto ${ }^{c}$, Lucio Martinez-Ramirez ${ }^{\mathrm{c}}$
}

\begin{abstract}
Background: The seroepidemiology of Leptospira infection in workers occupationally exposed to raw meat has been poorly studied. This work aimed to determine the association between Leptospira exposure and the occupation of meat worker, and to determine the seroprevalence association with socio-demographic, work, clinical and behavioral characteristics of the meat workers studied.
\end{abstract}

Methods: We performed a case-control study in 124 meat workers and 124 age- and gender-matched control subjects in Durango City, Mexico. Sera of cases and controls were analyzed for anti-Leptospira IgG antibodies using a commercially available enzyme immunoassay. Data of meat workers were obtained with the aid of a questionnaire. The association of Leptospira exposure with the characteristics of meat workers was analyzed by bivariate and multivariate analyses.

Results: Anti-Leptospira IgG antibodies were found in $22(17.7 \%)$ of 124 meat workers and in eight $(6.5 \%)$ of 124 controls $(\mathrm{OR}=3.12$; 95\% CI: 1.33 - 7.33; P = 0.006). Seroprevalence of Leptospira infection was similar between male butchers (17.6\%) and female butchers $(18.2 \%)(\mathrm{P}=1.00)$. Multivariate analysis of socio-demographic, work and behavioral variables showed that Leptospira exposure was associated with duration in the activity, rural residence, and consumption of snake meat and unwashed raw fruits.

Conclusions: This is the first case-control study of the association of Leptospira exposure with the occupation of meat worker. Results indicate that meat workers represent a risk group for Leptospira exposure. Risk factors for Leptospira exposure found in this study may help in the design of optimal preventive measures against Leptospira infection.

Keywords: Leptospira; Case-control study; Epidemiology; Seroprevalence; Meat workers; Mexico

Manuscript accepted for publication January 12, 2016

aBiomedical Research Laboratory, Faculty of Medicine and Nutrition, Juarez University of Durango State, Durango, Mexico

bInstitute for Scientific Research "Dr. Roberto Rivera Damm", Juarez University of Durango State, Durango, Mexico

${ }^{\mathrm{c} C l i n i c a}$ de Medicina Familiar, Instituto de Seguridad y Servicios Sociales de los Trabajadores del Estado, Predio Canoas S/N, 34079 Durango, Mexico

${ }^{\mathrm{d} C}$ Corresponding Author: Cosme Alvarado-Esquivel, Faculty of Medicine and Nutrition. Av. Universidad S/N, 34000 Durango, Dgo, Mexico.

Email: alvaradocosme@yahoo.com

doi: http://dx.doi.org/10.14740/jocmr2463w

\section{Introduction}

Bacteria of the genus Leptospira cause a disease known as leptospirosis [1]. This disease is a worldwide zoonosis [2,3]. Leptospira is excreted in the urine of infected animals [1]. Humans become infected with Leptospira by direct or indirect contact with infected animals and their urine or by contact with contaminated water and soil [1]. A number of animals can be infected with Leptospira, i.e., swine [2], sheep [4, 5], cows [1], rats, dogs [1, 6], and horses [7]. Leptospira infection does not only occur in domestic animals but also in wild and peri-domestic animals including deer [8] and other mammals, birds, and reptiles [9]. Infection with Leptospira is usually asymptomatic [10]. Clinical manifestations of leptospirosis include influenza-like symptoms, pulmonary hemorrhage [11], and renal and liver failure [12]. Leptospira infection in pregnant women may lead to fetal and maternal morbidity and mortality [13].

Very little is known about the seroepidemiology of Leptospira infection in workers occupationally exposed to raw meat. Studies in New Zealand have revealed seroprevalences of Leptospira infection of $10.2 \%$ in meat inspectors [14], and $6.2 \%$ in meat workers [15]. In a study in Italy, researchers found an $11.76 \%$ seroprevalence of Leptospira infection in meat workers [16], whereas in a study in Tanzania, abattoir workers had a $17.1 \%$ seroprevalence of Leptospira infection [17]. To the best of our knowledge, there is no case-control study about the association of Leptospira infection with the occupation of meat worker. In addition, we are not aware of any survey about Leptospira infection in meat workers in Mexico. Therefore, we sought to determine the association of Leptospira exposure with the occupation of meat worker in Durango City, Mexico and to determine the socio-demographic, clinical, work and behavioral characteristics of meat workers associated with Leptospira exposure.

\section{Materials and Methods}

\section{Workers occupationally exposed to raw meat and controls}

We performed an age- and gender-matched case-control study using serum samples from recent studies about the seroepidemiology of Toxoplasma gondii infection in Durango City, Mexico [18, 19]. Cases included 124 meat workers, and controls included 124 subjects without an occupation of meat worker. Sera from all participants were analyzed for the presence of anti-Leptospira IgG antibodies. Meat workers includ- 
ed in the study were those who have worked as butchers in abattoirs or butcher's shops for at least 6 months, aged 16 years and older, and who accepted to participate in the study. None of the following characteristics of meat workers was a restrictive criterion for enrollment: gender, socio-economic status, or educational level. Fifty-nine meat workers were enrolled in 35 private butcher's shops, 35 in a federal abattoir and 30 in a municipal abattoir. Meat workers (21 females and 103 males) were aged $16-71$ years old (mean $38.5 \pm 13.2$ years). Controls were randomly selected from the general population of $\mathrm{Du}$ rango City. They had occupations other than meat worker and were matched with cases by age and gender. We included one control for each case. The control group included 124 subjects (21 females and 103 males) aged $16-72$ years (mean: $38.85 \pm$ 13.68 years). The mean age in controls was comparable to that in meat workers $(\mathrm{P}=0.69)$.

\section{Characteristics of meat workers}

Socio-demographic, clinical, work and behavioral data of meat workers were obtained from previously submitted questionnaires [18]. Socio-demographic data included age, gender, birthplace, residence, educational level, and socio-economic status. Clinical data were current suffering from any disease, history of blood transfusion, and presence of visual impairment. Work data included duration (years) in the activity, frequency of contact with raw meat, habitual use of safety practices (use of hand gloves, face masks, and glasses), history of splashes at face with blood or raw meat, injuries with sharp material at work, and eating when working. Behavioral data were raising farm animals, foreign traveling, consumption of meat (pork, beef, goat, lamb, boar, chicken, turkey, pigeon, duck, rabbit, venison, squirrel, horse, opossum, snake or other), consumption of raw or undercooked meat, untreated water, unwashed raw vegetables and fruits, and soil contact.

\section{Detection of anti-Leptospira IgG antibody}

Stored serum samples from cases and controls were analyzed for anti-Leptospira IgG antibodies by a commercially available enzyme immunoassay "Leptospira IgG ELISA test" kit (Diagnostic Automation Inc., Calabasas, CA). Seroreactivity to anti-Leptospira IgG antibodies was considered when an absorbance reading equal to or higher than 0.5 optical density (OD) units in a serum sample was found. Weak and strong seroreactivities of samples were considered when absorbance readings between 0.5 and $1.0 \mathrm{OD}$ units and higher than $1.0 \mathrm{OD}$ were found, respectively. Immunoassays were performed following the manufacturer's instructions. According to the kit's insert, the immunoassay used has a sensitivity of $100 \%$ and a specificity of $100 \%$. Positive and negative controls were included in each run.

\section{Statistical analysis}

Results were analyzed with the software Epi Info version 7, and SPSS version 15.0 (SPSS Inc. Chicago, IL). For calculation of the sample size, we used a $95 \%$ confidence level, a power of $80 \%$, a 1:1 proportion of cases and controls, a reference seroprevalence of 5.6\% [20] as the expected frequency of exposure in controls, and an odds ratio (OR) of 3.5. The result of the sample size calculation was 117 cases and 117 controls. To compare age values among cases and controls, the paired Student's $t$-test was used. We used the Pearson's Chi-square test or the two-tailed Fisher's exact test (when cell values were less than 5) for comparison among groups. The association of Leptospira seropositivity with the socio-demographic, clinical, work and behavioral characteristics of meat workers was assessed by bivariate and multivariate analysis. Only variables with a $P$ value $\leq 0.05$ obtained in the bivariate analysis were included in the multivariate analysis. The Hosmer-Lemeshow goodness of fit test was used to assess the fitness of our regression model. OR and $95 \%$ confidence interval $(\mathrm{CI})$ were calculated by logistic regression with the Enter method. A P value $<$ 0.05 was considered statistically significant.

\section{Ethical aspects}

This study was approved by the Ethics Committee of the Instituto de Seguridad y Servicios Sociales de los Trabajadores del Estado in Durango City, Mexico.

\section{Results}

Anti-Leptospira IgG antibodies were found in $22(17.7 \%)$ of 124 meat workers and in eight $(6.5 \%)$ of 124 controls $(\mathrm{OR}=$ 3.12; $95 \%$ CI: $1.33-7.33$; $\mathrm{P}=0.006)$. High $(\mathrm{OD}>1.0)$ antiLeptospira IgG antibody levels were found in six (4.8\%) of 124 meat workers and in two $(1.6 \%)$ of 124 controls $(\mathrm{OR}=3.10$; $95 \%$ CI: 0.61 - 15.67; $\mathrm{P}=0.28)$. Seroprevalence results were stratified by gender. Anti-Leptospira IgG antibodies were found in four $(18.2 \%)$ of 22 female meat workers and in one $(4.5 \%)$ of 22 female controls $(\mathrm{OR}=4.66$; 95\% CI: $0.47-45.62 ; \mathrm{P}=$ $0.34)$. Whereas anti-Leptospira $\operatorname{IgG}$ antibodies were found in $18(17.6 \%)$ of 102 male meat workers and in seven $(6.9 \%)$ of 102 male controls $(\mathrm{OR}=2.90 ; 95 \% \mathrm{CI}: 1.15-7.30 ; \mathrm{P}=0.01)$. Seroprevalence of Leptospira infection was similar between male butchers $(17.6 \%)$ and female butchers $(18.2 \%)(\mathrm{P}=1.00)$.

Concerning the socio-demographic, clinical, work and behavioral characteristics in the meat workers studied, the variables duration in the activity, residence area, and consumption of snake meat and unwashed raw fruits had $\mathrm{P}$ values $<0.05$ by bivariate analysis. Other socio-demographic and behavioral characteristics including age, gender, birthplace, educational level, socio-economic status, raising farm animals, traveling, consumption of meat other than snake meat, consumption of raw or undercooked meat, untreated water, unwashed raw vegetables, and soil contact had $\mathrm{P}$ values $\geq 0.05$ by bivariate analysis. None of the clinical characteristics studied including presence of any disease, history of blood transfusion, and presence of visual impairment had $\mathrm{P}$ values $<0.05$ by bivariate analysis. In addition, other work characteristics, i.e., frequency of con- 
Table 1. Bivariate Analysis of a Selection of Characteristics of Meat Workers and Seroprevalence of Leptospira Exposure

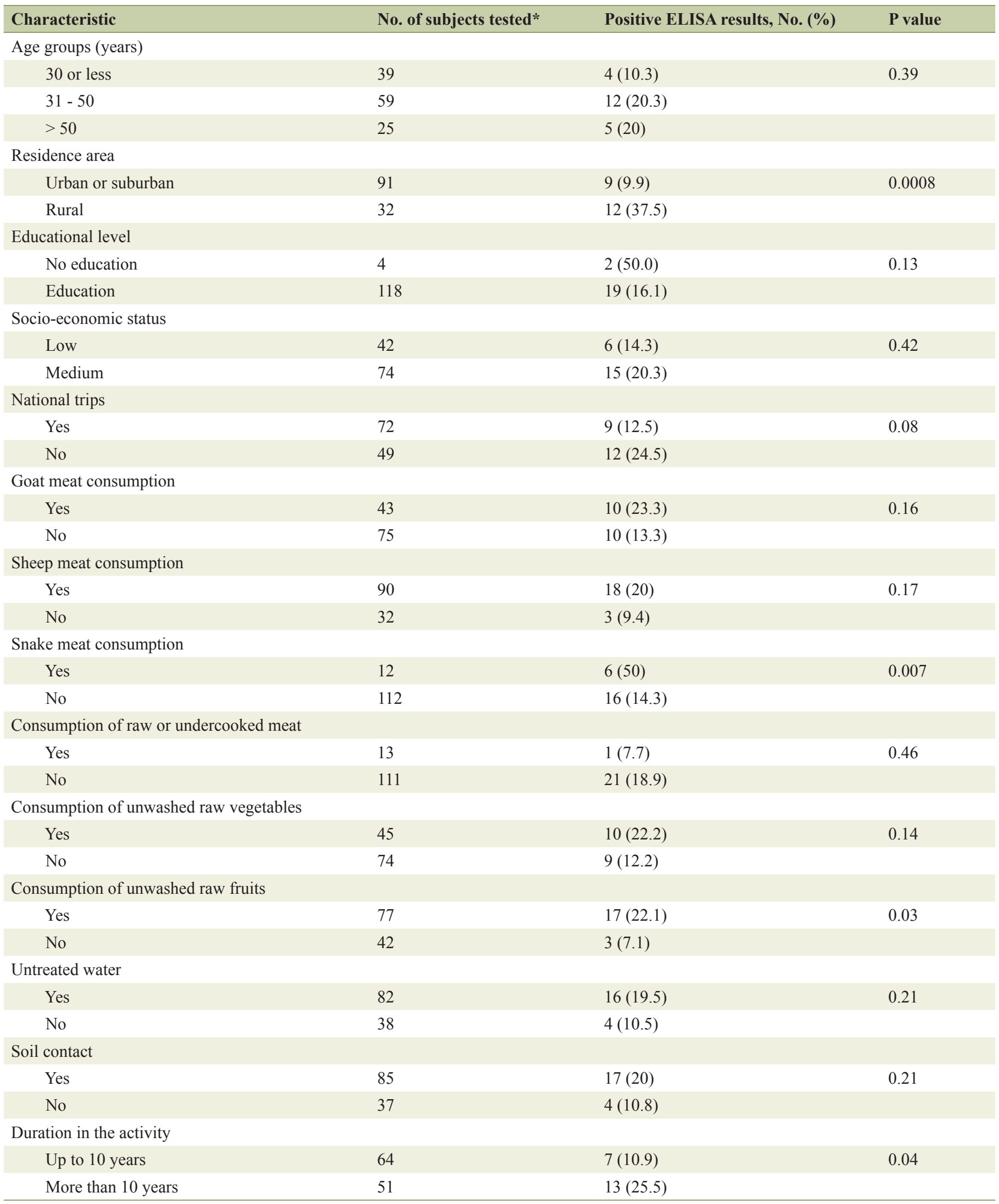


Table 1. Bivariate Analysis of a Selection of Characteristics of Meat Workers and Seroprevalence of Leptospira Exposure - (continued)

\begin{tabular}{llll}
\hline Characteristic & No. of subjects tested* & Positive ELISA results, No. (\%) & P value \\
\hline $\begin{array}{l}\text { Safety practices (gloves, mask, glasses) } \\
\quad \text { Yes }\end{array}$ & 11 & $4(36.4)$ & 0.11 \\
$\quad$ No & 105 & $17(16.2)$ & 0.15 \\
$\begin{array}{l}\text { Splash of blood or raw meat at face } \\
\quad \text { Yes }\end{array}$ & 88 & $18(20.5)$ & 0.65 \\
$\quad$ No & 32 & $3(9.4)$ & \\
$\begin{array}{l}\text { Injuries at work } \\
\quad \text { Yes }\end{array}$ & 111 & $19(17.1)$ & 0.16 \\
$\quad$ No & 9 & $2(22.2)$ & \\
Visual impairment & & $16(20)$ & $4(10)$ \\
$\quad$ Yes & 80 & 40 & \\
$\quad$ No &
\end{tabular}

*Sums may not add up to 124 because of some missing values.

tact with raw meat, habitual use of safety practices, history of splashes at face with blood or raw meat, injuries with sharp material at work, and eating when working had $\mathrm{P}$ values $<0.05$ by bivariate analysis. A correlation of the seroprevalence of Leptospira infection with a selection of characteristics of meat workers is shown in Table 1. Multivariate analysis of sociodemographic, work and behavioral variables with $\mathrm{P}$ values $<$ 0.05 obtained by bivariate analysis showed that Leptospira exposure was positively associated with duration in the activity, rural residence, and consumption of snake meat and unwashed raw fruits. Table 2 shows the results of the multivariate analysis. The result of the Hosmer-Lemeshow test $(\mathrm{P}=0.17)$ suggested a good fitting of our regression model.

\section{Discussion}

Surveys of Leptospira infection in workers with occupational exposure to raw meat have been performed in a limited number of countries. In addition, the magnitude of the association of Leptospira seropositivity with the occupation of meat worker has not been previously evaluated by an age- and gender-matched case-control study. Therefore, this study aimed to determine the association of Leptospira seropositivity and the occupation of meat worker in the northern Mexican city of Durango. We found that seroprevalence of Leptospira expo- sure was significantly higher in meat workers than in control subjects. Male and female meat workers had a similar seroprevalence of Leptospira exposure. The seroprevalence found in meat workers (17.7\%) is also higher than the $4.4 \%$ seroprevalence found in waste pickers in the same Durango City [20]. On the other hand, the seroprevalence found in meat workers is comparable with the $15.6 \%$ seroprevalence reported in the general population in rural Durango [21]. In an international context, the seroprevalence found in meat workers in Durango is higher than the $10.2 \%$ seroprevalence reported in meat inspectors in New Zealand [14], the $11.76 \%$ seroprevalence found in meat workers in Italy [16], and the 7\% seroprevalence reported in slaughterhouse workers in Colombia [22]. The seroprevalence found in this study is comparable with the $17.1 \%$ found in abattoir workers in Tanzania [17]. In contrast, the seroprevalence found in meat workers in Durango is lower than the 29.2\% seroprevalence of Leptospira infection found in butchers/abattoir workers in Nigeria [23].

We searched for factors associated with Leptospira infection in meat workers. Interestingly, multivariate analysis showed that Leptospira exposure was associated with duration in the activity of meat worker. This finding is consistent with that found in a recent study in New Zealand where researchers found that the time worked in the meat industry was a risk factor for Leptospira infection [24]. Multivariate analysis also showed that Leptospira exposure was associated with the fol-

Table 2. Results of the Multivariate Regression Analysis

\begin{tabular}{llll} 
Variables & P value & Odds ratio & $\mathbf{9 5 \%}$ confidence interval \\
\hline Rural residence & 0.01 & 4.29 & $1.29-14.20$ \\
Duration in the activity & 0.03 & 3.87 & $1.13-13.22$ \\
Snake meat consumption & 0.01 & 7.20 & $1.48-34.91$ \\
Consumption of unwashed raw fruits & 0.03 & 6.68 & $1.16-38.55$ \\
\hline
\end{tabular}


lowing factors: rural residence, and consumption of snake meat and unwashed raw fruits. We have recently studied the seroepidemiology of Leptospira infection in the general population in rural Durango and found a high $(15.6 \%)$ seroprevalence of Leptospira infection [19]. However, meat workers with rural residence had a more than two-fold higher seroprevalence $(37.5 \%)$ of Leptospira infection than the one reported in the general population in rural Durango. This fact suggests that other factors, i.e., contact with raw meat might have contributed to the increasing seroprevalence. It is likely that people living in rural communities have more contact with animals than people do in urban communities. In fact, rural work (raising animals and agriculture) was a risk factor for Leptospira seropositivity in a survey in Colombia [22]. Poor education of the head of the family was a risk factor for Leptospira infection in rural Durango [21]. Intriguingly, Leptospira seropositivity was associated with consumption of snake meat. To the best of our knowledge, there is no previous report of this association. The epidemiological link of Leptospira infection and consumption of snake meat is supported by the fact that infection with Leptospira has been demonstrated in snakes. Seroreactivity to several Leptospira species was found in six of 22 snakes sampled in Slovenia [25]. In addition, seropositivity to Leptospira was found in 13 of 18 snakes in Argentina [26]. In contrast, none of 10 free-ranging Venezuelan anacondas had anti-Leptospira antibodies [27]. In a study in Brazil, researchers found not only antibodies to Leptospira in snakes but also found DNA of Leptospira by polymerase chain reaction in kidney and liver of snakes [28]. Further studies to confirm the association of Leptospira infection and consumption of snake meat are needed. In our study, Leptospira exposure was associated with consumption of unwashed raw fruit. This finding reflects poor hygiene practices. Fruits may be contaminated with Leptospira when contact soil contaminated with urine of Leptospira-infected rodents and other animals. In fact, fruits have been related with leptospirosis. A case of leptospirosis with acute renal failure in a man who ate unwashed raw fruits was reported [29]. A case of Weil's syndrome with bone marrow involvement in a 65-year-old man who in recent weeks had collected walnuts in a region with infestation of rats was reported in Germany [30]. Further studies on the association of Leptospira infection and consumption of unwashed raw fruits are needed.

\section{Conclusions}

This is the first case-control study of the association of Leptospira exposure with the occupation of meat worker, and of the association of Leptospira exposure with the consumption of snake meat. Results indicate that meat workers represent a risk group for Leptospira exposure. Risk factors for Leptospira exposure found in this study may help in the design of optimal preventive measures against Leptospira infection.

\section{Conflict of Interest}

The authors declare that they have no conflict of interest.

\section{Financial Support}

This work was financially supported by Juarez University of Durango State, Mexico.

\section{References}

1. Samir A, Soliman R, El-Hariri M, Abdel-Moein K, Hatem ME. Leptospirosis in animals and human contacts in Egypt: broad range surveillance. Rev Soc Bras Med Trop. 2015;48(3):272-277.

2. Colavita G, Paoletti M. [Leptospirosis: occupational risk in the chain of food of animal origin]. G Ital Med Lav Ergon. 2007;29(1):21-24.

3. Gamage CD, Koizumi N, Perera AK, Muto M, NwaforOkoli C, Ranasinghe S, Kularatne SA, et al. Carrier status of leptospirosis among cattle in Sri Lanka: a zoonotic threat to public health. Transbound Emerg Dis. 2014;61(1):91-96.

4. Dorjee S, Heuer C, Jackson R, West DM, Collins-Emerson JM, Midwinter AC, Ridler AL. Prevalence of pathogenic Leptospira spp. in sheep in a sheep-only abattoir in New Zealand. N Z Vet J. 2008;56(4):164-170.

5. Barbante P, Shimabukuro FH, Langoni H, Richini-Pereira VB, Lucheis SB. Leptospira spp. infection in sheep herds in southeast Brazil. J Venom Anim Toxins Incl Trop Dis. 2014;20:20.

6. Suepaul SM, Carrington CV, Campbell M, Borde G, Adesiyun AA. Seroepidemiology of leptospirosis in dogs and rats in Trinidad. Trop Biomed. 2014;31(4):853-861.

7. Verma A, Stevenson B, Adler B. Leptospirosis in horses. Vet Microbiol. 2013;167(1-2):61-66.

8. Andreoli E, Radaelli E, Bertoletti I, Bianchi A, Scanziani E, Tagliabue S, Mattiello S. Leptospira spp. infection in wild ruminants: a survey in Central Italian Alps. Vet Ital. 2014;50(4):285-291.

9. Jobbins SE, Alexander KA. Evidence of Leptospira sp. infection among a diversity of African wildlife species: beyond the usual suspects. Trans R Soc Trop Med Hyg. 2015;109(5):349-351.

10. Silva HR, Tavares-Neto J, Bina JC, Meyer R. [Leptospiral infection and subclinical presentation among children in Salvador, Bahia]. Rev Soc Bras Med Trop. 2003;36(2):227-233.

11. Hu W, Lin X, Yan J. Leptospira and leptospirosis in China. Curr Opin Infect Dis. 2014;27(5):432-436.

12. Forbes AE, Zochowski WJ, Dubrey SW, Sivaprakasam V. Leptospirosis and Weil's disease in the UK. QJM. 2012;105(12):1151-1162.

13. Puliyath G, Singh S. Leptospirosis in pregnancy. Eur $\mathbf{J}$ Clin Microbiol Infect Dis. 2012;31(10):2491-2496.

14. Blackmore DK, Bell L, Schollum L. Leptospirosis in meat inspectors: preliminary results of a serological survey. N Z Med J. 1979;90(648):415-418.

15. Blackmore DK, Schollum L. The occupational hazards of leptospirosis in the meat industry. N Z Med J. 1982;95(712):494-497. 
16. Crevatin D, Banfi E, Crotti D, Ruaro E, Cinco M. Serosurvey on the presence of leptospiral agglutinins in humans in Northern Italy. Eur J Epidemiol. 1986;2(1):4447.

17. Schoonman L, Swai ES. Risk factors associated with the seroprevalence of leptospirosis, amongst at-risk groups in and around Tanga city, Tanzania. Ann Trop Med Parasitol. 2009;103(8):711-718.

18. Alvarado-Esquivel C, Liesenfeld O, Estrada-Martinez S, Felix-Huerta J. Toxoplasma gondii infection in workers occupationally exposed to raw meat. Occup Med (Lond). 2011;61(4):265-269.

19. Alvarado-Esquivel C, Estrada-Martinez S, Pizarro-Villalobos H, Arce-Quinones M, Liesenfeld O, Dubey JP. Seroepidemiology of Toxoplasma gondii infection in general population in a northern Mexican city. J Parasitol. 2011;97(1):40-43.

20. Alvarado-Esquivel C, Hernandez-Tinoco J, Sanchez-Anguiano LF, Ramos-Nevarez A, Cerrillo-Soto SM, GuidoArreola CA. Leptospira Exposure and Waste Pickers: A Case-Control Seroprevalence Study in Durango, Mexico. J Clin Med Res. 2015;7(8):637-640.

21. Alvarado-Esquivel C, Sanchez-Anguiano LF, HernandezTinoco J. Seroepidemiology of Leptospira Exposure in General Population in Rural Durango, Mexico. Biomed Res Int. 2015;2015:460578.

22. Gongora A, Parra J, Aponte L, Gomez L. [Seroprevalence of Leptospira spp in population groups of Villavicencio, Colombia]. Rev Salud Publica (Bogota). 2008;10(2):269278.
23. Onyemelukwe NF. A serological survey for leptospirosis in the Enugu area of eastern Nigeria among people at occupational risk. J Trop Med Hyg. 1993;96(5):301-304.

24. Dreyfus A, Wilson P, Collins-Emerson J, Benschop J, Moore S, Heuer C. Risk factors for new infection with Leptospira in meat workers in New Zealand. Occup Environ Med. 2015;72(3):219-225.

25. Lindtner-Knific R, Vergles-Rataj A, Vlahovic K, Zrimsek P, Dove A. Prevalence of antibodies against Leptospira sp. in snakes, lizards and turtles in Slovenia. Acta Vet Scand. 2013;55:65

26. Stanchi NO, Grisolia CS, Martino PE, Peluso FO. [Presence of antileptospira antibodies in ophidia in Argentina]. Rev Argent Microbiol. 1986;18(3-4):127-130.

27. Calle PP, Rivas J, Munoz M, Thorbjarnarson J, Holmstrom W, Karesh WB. Infectious disease serologic survey in free-ranging Venezuelan anacondas (Eunectes murinus). J Zoo Wildl Med. 2001;32(3):320-323.

28. Biscola, Natalia P, Fornazari, Felipe, Saad, Eduardo, Richini-Pereira, Virginia B, Campagner, Michelle V, Langoni, Helio, Barraviera, Benedito, Ferreira Junior, Rui S. Serological investigation and PCR in detection of pathogenic leptospires in snakes. Pesquisa Veterinaria Brasileira. 2011;31(9):806-811.

29. Cestero-Rivera R, Ramirez-Rivera J, Jaume-Anselmi F, Guerrero-Dujarric O. Acute renal failure in a man with a small farm. Bol Asoc Med P R. 2006;98(2):114-116.

30. Wenz M, Gorissen B, Wieshammer S. [Weil's syndrome with bone marrow involvement after collecting walnuts]. Dtsch Med Wochenschr. 2001;126(41):1132-1135. 